\title{
Contralateral Body Half Hypalgesia in a Patient with Lateral Medullary Infarction: Atypical Wallenberg Syndrome
}

\author{
She-Qing Zhang ${ }^{\mathrm{a}}$ Ming-Yuan Liu ${ }^{\mathrm{a}}$ Bo Wan ${ }^{\mathrm{b}}$ Hui-Min Zheng ${ }^{\mathrm{a}}$ \\ ${ }^{\mathrm{a}}$ Department of Neurology, Changhai Hospital, and ${ }^{\mathrm{b}}$ State Key Laboratory of Genetic Engineering, \\ Fudan University, Shanghai, China
}

\begin{abstract}
Dear Sir,
Lateral medullary infarction is one of the most frequently encountered cerebral infarctions involving the brainstem and often manifests as Wallenberg syndrome. Typically, it presents with vertigo, hoarseness and dysphagia, Horner's syndrome and cerebellar ataxia on the same side of the lesion, and hypalgesia [1], which usually involves the ipsilateral face and the opposite trunk and limbs (crossed sensory symptoms). Hypalgesia that involves the contralateral face, trunk, arm and leg without affecting the ipsilateral side of the face has seldom been reported [2]. Recently, we have encountered a female patient, who suddenly experienced hypesthesia in the entire contralateral body half with the ipsilateral side of the face unaffected.
\end{abstract}

\section{Case Report}

A 44-year-old woman patient suddenly developed dizziness, which disappeared gradually in half an hour. An hour later, she felt dysarthria and dysphagia, and then noticed paresthesia and numbness on the entire right side of the body, especially in the right trunk and limbs. The following morning, she felt lateropulsion to the left when walking. She did not complain of vertigo, diplopia or vomiting.
On admission she was normotensive, and her general examination was normal. Neurological examination showed left Horner's syndrome, with the left eye mildly ptotic (fig. 1a). There was no nystagmus in both eyes. The left pupil was about $3 \mathrm{~mm}$ and the right one $4 \mathrm{~mm}$ in diameter; both pupils were alert to light. The left palate was paraparetic, and the left throat reflex was decreased. The tongue moved well and there was no facial weakness. Sensation to pain and heat was decreased substantially in the right trunk and limbs, but decreased mildly in the right face, and normal in the left face and the left body. Bilateral vibratory and postural sensation was preserved. The left finger-nose test and the heel-knee-shin test were minimally incoordinated, compared with those of the right. When standing upright and closing the eyes, she felt mild lateropulsion to the left. There was no weakness of the limbs.

Cerebral MRI detected a small bandlike ischemic lesion in the left retro-olivary midlateral tegmentum, with preservation of the far dorsal vestibular nucleus and inferior cerebellar peduncle (fig. 1c). Angiographic sequences disclosed congenital dysplasia of the left vertebral artery and segmental stenosis at the origin of the posterior inferior cerebellar artery (fig. 1b).

\section{Discussion}

Symptoms and signs found in patients with lateral medullary infarction depend on the dorsal-ventral, medial-lateral and retrocaudal locations of the lesion. Vestibulocerebellar symptoms and signs are nearly always present in these patients [3]. The present patient presented with left Horner's syndrome, dysphagia, mild hoarseness and mild lateropulsion to the left. These clinical findings, together with the radiological findings of the left medulla, confirmed the diagnosis of lateral medullary infarction without any doubt. What was extraordinary in this patient was that she merely felt dizziness, which gradually disappeared in half an hour after onset. In addition, she did not feel obvious vertigo, and the left finger-nose test and the heelknee-shin test were only minimally incoordinated, indicating that the infarction did not involve the vestibular nucleus and the left inferior cerebellar peduncle. This condition was later explained by the MRI findings, which disclosed normal signals at the dorsal edge of the medulla. It is common knowledge that the vestibular nucleus is located far dorsally to the bottom of the fourth ventricle. This type of infarction without involvement of the vestibular nucleus and inferior cerebellar peduncle is a rare condition.

\section{KARGER \\ Fax +41 613061234 \\ E-Mail karger@karger.ch}

www.karger.com
She-Qing Zhang, MD

Department of Neurology

Changhai Hospital, No. 174 Changhai Road

Shanghai 200433 (China)

Tel. +8621 2507 2791, Fax +86216564 3250, E-Mail genplezhang@126.com 

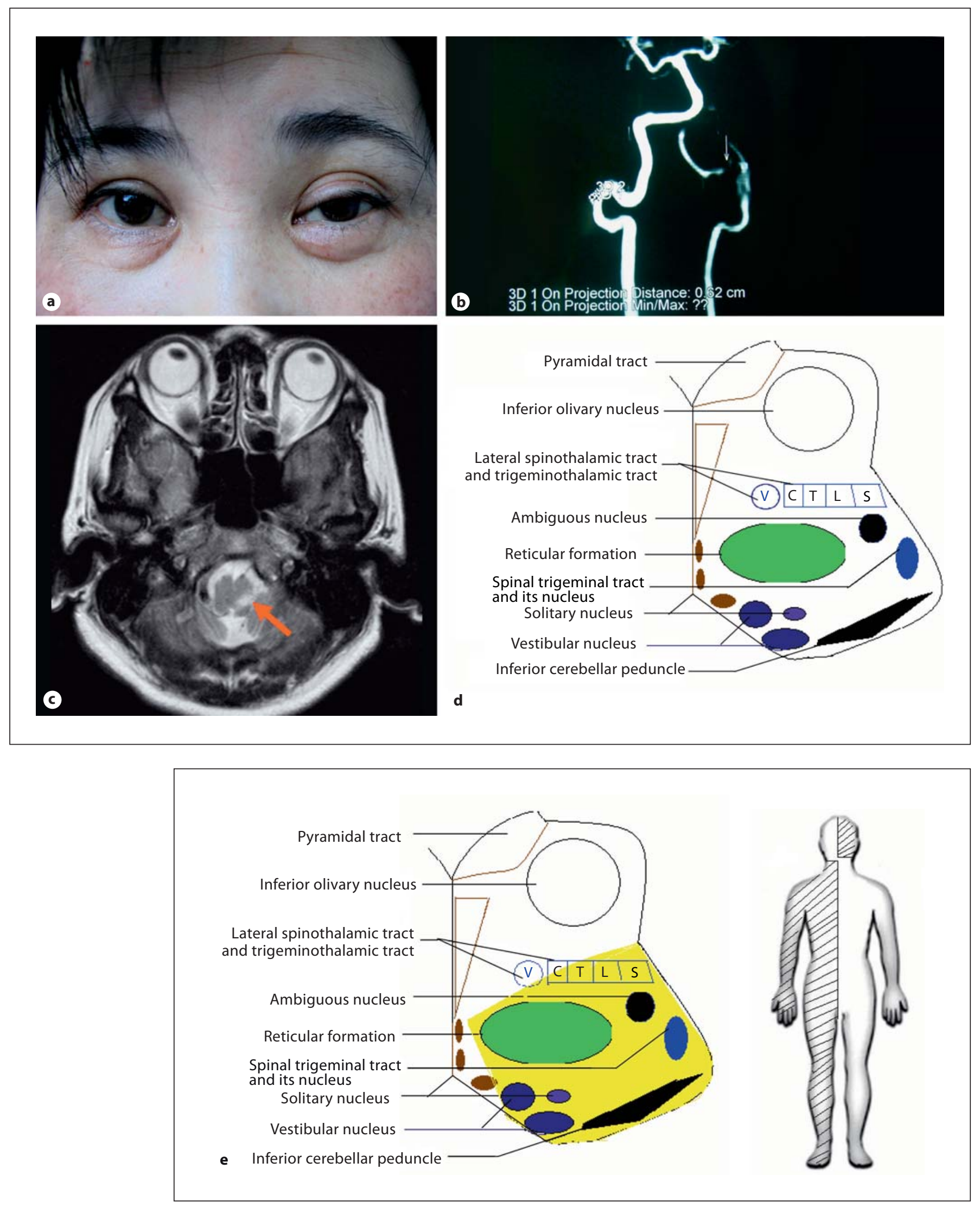

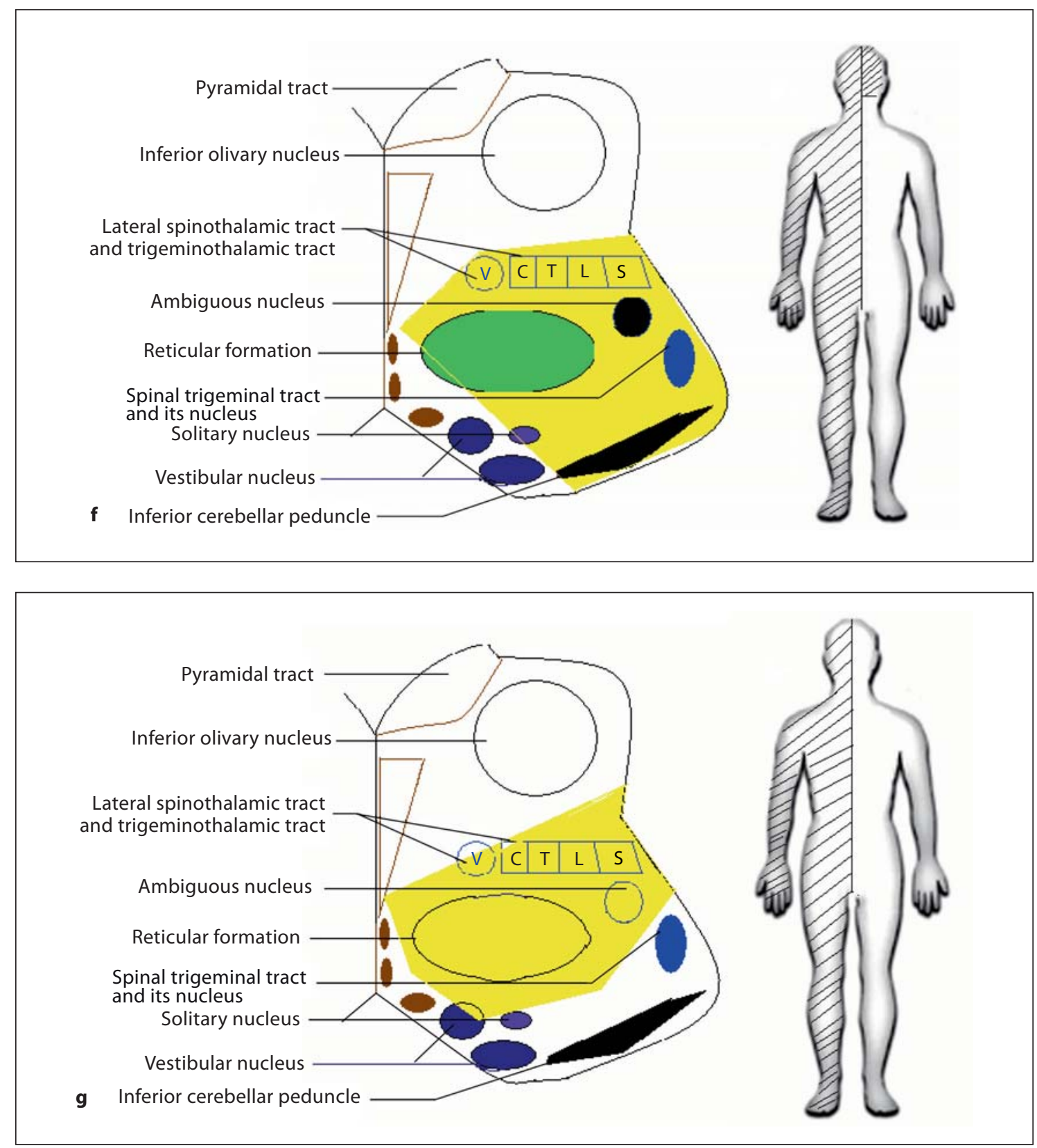

Fig 1. a Left Horner's syndrome. b Congenital dysplasia of the left vertebral artery and stenosis at the origin of the posterior inferior cerebellar artery. $\mathbf{c} \mathrm{T}_{2}$-weighted MRI shows hypersignals (red arrow) in the left midlateral medulla. d Diagram of normal medullary structure showing the somatotopical organization of the lateral spinothalamic tract and ventral trigeminothalamic tract. e Far dorsal lateral medullary infarction (yellow) and its related crossed sensory defect: type I. f Enlarged dorsal lateral infarction (yellow), including the ventral trigeminothalamic tract, and its related crossed sensory defect: type II. g Midlateral medullary infarction (yellow) and its related contralateral hypalgesia like that in our case: type III.

Moreover, the characteristic crossed sensory presentations including hypalgesia in the ipsilateral face and the contralateral trunk and limbs were not seen. This classic pattern of sensory symptoms (type I) is due to the involvement of the crossed lateral spinothalamic tract and the ipsilateral descending spinal trigeminal tract and its nucleus $[4,7]$. This crossed type corresponds to far dorsal lateral medullary lesions (fig. 1e). If the lesion extends more ventrally and medially, the crossed trigeminothalamic tract (which carries pain and heat sensation from the contralateral side of the face) may be involved, and the patient may 

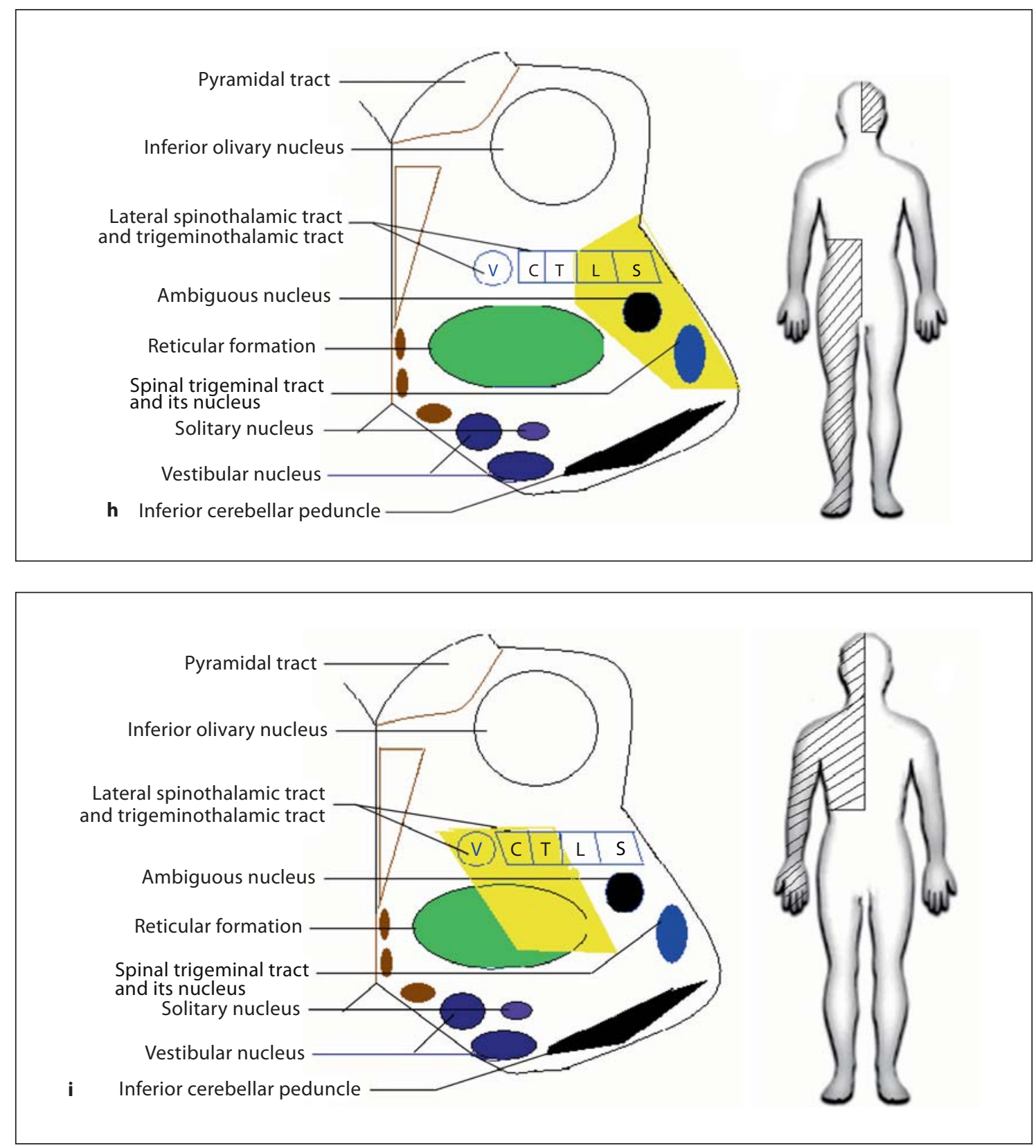

Fig. 1. h Far lateral infarction (yellow) and its partially crossed sensory defect: type IV. i Restricted mediolateral infarction (yellow) and its related contralateral partial sensory defect: type V.

present with hypalgesia in the whole contralateral body, including the face, trunk and limbs, in addition to the ipsilateral face (fig. 1f); this type of sensory defect is type II. In our patient, however, the ipsilateral descending spinal trigeminal tract and its nucleus (which lies more dorsally to the spinothalamic tract) had obviously been spared, and the ventral crossed trigemino- thalamic tract had been involved (fig. $1 \mathrm{~g}$ ). This is why our patient did not feel numbness or hypesthesia in the left face and why the left corneal reflex was sensitive. This type of sensory defect that only involves the contralateral body is type III.

Still, there are other less common sensory defect patterns in lateral medullary infarction, which have been clearly de- scribed by Matsumoto et al. [6]. One pattern presents with hypalgesia in the ipsilateral face and contralateral lower trunk and leg (type IV). This type of sensory defect is caused by the infarction of far lateral location, which only involves the lateral descending spinal trigeminal tract and its nucleus, and the ventral lateral part of the spinal thalamic tract (fig. 1h). An- 
other pattern of sensory defect manifests hypalgesia only in the contralateral face, arm and upper trunk, without involvement of the ipsilateral face. This rare type of sensory defect (type $\mathrm{V}$ ) is caused by restricted infarction of the mediolateral medulla, which only involves the crossed trigeminal thalamic tract and the medial part of the spinal thalamic tract (fig. 1i) [5, 6]. These partial sensory defects on the contralateral side of the body are due to somatotopical organization of the spinothalamic tract (fig. 1d). The sacral afferent fibers are located in the lateral medullary part, and the cervical afferent fibers ascend more medially. The crossed ventral trigeminothalamic tract appends on the medial part of the spinothalamic tract and carries pain and heat sensation from the contralateral side of the face [8].

In conclusion, we report a rare type of lateral medullary infarction, which presents without obvious vertigo, and the sensory defect is entirely in the contralateral body half.

\section{References}

1 Ropper AH, Brown RH: Adams and Victor's Principles of Neurology, ed 8. New York, McGraw-Hill, 2005, pp 678-679.

2 Vuadens P, Bogousslavsky J: Face-armtrunk-leg sensory loss limited to the contralateral side in lateral medully infarction: a new variant. J Neurol Neurosurg Psychiatry 1998;65:255-257.
3 Asbury AK, Mckhann GM, McDonald WI, et al: Diseases of the Nervous System, ed 3. Cambridge, Cambridge University Press, 2002, pp 1350-1352.

4 Stopford JSB: Arteries of pons and medulla oblongata. J Anat 1924;59:120-128.

5 Soffin G, Feldman M, Bender MB: Alteration of sensory levels in vascular lesions of lateral medulla. Arch Neurol 1968;18:178-190.

6 Matsumoto S, Okuda B, Imai T, et al: A sensory level on the trunk in lower lateral brainstem lesion. Neurology 1988,38:1515-1519.

7 Brodal A: Neurological Anatomy in Relation to Clinical Medicine. Oxford, Oxford University Press, 1981, pp 141-143.

8 Vuileumier P, Bogousslavsky J, Regh F: Infarction of the lower brainstem: clinical, aetiological and MRI topographical correlations. Brain 1995;118:1013-1025. 